Www.jmscr.igmpublication.org

Index Copernicus Value: 79.54

ISSN (e)-2347-176x ISSN (p) 2455-0450

crossref DOI: https://dx.doi.org/10.18535/jmscr/v7i3.223

\title{
Arrhythmia in Case of Celphos Poisoning: A Rare Case Report
}

\author{
Authors \\ Dr Ashiti Jain ${ }^{1}$, Dr Saurabh Goel ${ }^{2}$, Dr Sandeep Rai ${ }^{3}$ \\ ${ }^{1}$ Junior Resident, ${ }^{2}$ Senior Resident, ${ }^{3}$ Professor \\ Dept of Internal Medicine, MGM Medical College and Hospital, Navi Mumbai
}

\begin{abstract}
Celphos poisoning is extremely lethal and invariably suicidal in nature, owing to the release of phosphine gas, which inhibits electron transport chain and thereby myocardial suppression. Celfos is known to cause varying electric abnormalities of heart, the most frequent arrhythmia being atrial fibrillation followed by ventricular fibrillation, sustained $A V$ Conduction defect leading to $2^{\text {nd }}$ and $3^{\text {rd }}$ degree AV block. We hereby report a case of celphos poisoning presenting with atrial fibrillation. ${ }^{(1)}$
\end{abstract}

\section{Introduction}

Celphos (aluminium phosphide) is one of the most common suicidal poisoning agent, easily bought over the counter. Its high toxicity and absence of specific antidote results in very high mortality, especially in north, west and central INDIA. Its toxicity results from the release of phosphine gas when the tablet comes in contact with moisture and gastrointestinal tract. Phosphine gas primarily affects heart, gastrointestinal tract, lungs, and the kidneys. ${ }^{(2)}$

\section{Case Report}

30 year old female presented in ER with multiple episodes of vomiting after consumption of 8 fresh tabs of celphos(aluminium phosphide) in order to end her life. Patient presented in drowsy restless state with excruciating epigastric pain, sweating and difficulty in breathing. No past history of any major disease or psychiatric illness or any suicidal tendencies. The physical examination revealed a distressed patient with low volume irregular pulse of 130/min, BP of 90/60mmhg, RR 40/min, cold sweaty skin with saturation of $86 \%$ on room air. Abdominal examination revealed minimal guarding. CVS examination revealed tachycardia. Respiratory and neurological systems were normal., Gastric lavage with potassium permanganate was given immediately. Multi dose activated charcoal was given through ryles tube. Initial fluid challenge with $2 \mathrm{~L}$ of normal saline was given, to which patient did not respond and was started on ionotropic support. ECG revealed atrial fibrillation and patient being hemodynamically unstable, was given 200J of synchronised cardio version, thereby reverting to sinus rhythm. Due to decreased responsiveness, patient was intubated and put on mechanical ventilation. Inj magnesium sulphate $2 \mathrm{mg}$ was given, along with inj hydrocortisone, and broad spectrum antibiotics. ECG monitor later showed atrial fibrillation with fast ventricular rates, intravenous amiodarone was tried but with no beneficial effect. 
By the time blood investigations were available which showed normal renal and liver functions, with normal electrolytes. Blood picture revealed leucocytosis of 17230, hb 12.8, plt 3.66. Urine examination revealed proteinuria, trace blood, 1214 pus cells, 6-8 epithelial cells. TSH was 2.520. ABG analysis showed respiratory alkalosis with compensatory metabolic acidosis with lactate of 10.7. The patient later succumbed to profound shock and arrhythmia resistant to cardioversion.

\section{Discussion}

Aluminium phosphide, is a solid fumigant pesticide, widely used in India for storing cereal grains, available as Quickphos, Celphos, rice tablet. Each tablet weighs $3 \mathrm{gm}$ and releases $1 \mathrm{gm}$ of phosphine gas which has high dissolution and diffusion capacities. Mortality depends on whether the tablets are old exposed or the fresh ones opened from new packets, thereby increasing it from $30 \%$ to $100 \%$. The specified fatal dose is $0.15-0.5 \mathrm{gm}$. However most of the patients present with ingestion of three or more tablets which invariably results in death. The presence phosphine gas in gastric aspirate is confirmed by silver nitrate test.

Phosphine gas causes non competitive inhibition of cytochrome $\mathrm{C}$ oxidase thereby inhibiting mitochondrial oxidative phosphorylation leading to cellular hypoxia. It also causes focal myocardial necrosis resulting in transmembranal exchange of ions causing arrhythmias and myocardial suppression. ${ }^{(2)}$ Refractory myocardial depression carries a very high mortality. Cardiotoxicity is manifested as decreased LVEF. ECG changes vary from ST elevation/ depression, PR prolongation, broad QRS complexes, and right or left bundle branch block, supraventricular ectopics or fibrillation. Amiodarone can be safely used in dysrrhythmias in such cases. Vascular changes lead to marked low blood pressure, which do not respond to pressor agents. Profound shock resistant to vasopressors along with myocarditis, dysrrhythmias is the cause of death in aluminium phosphide poisoning. ${ }^{(3)}$
The aim of therapy is to sustain life of patient till phosphine gets excreted from the body. Antacids $60 \mathrm{ml}$ per hour helps to reduce phosphine absorption. THERE IS NO ANTIDOTE TO PHOSPHINE. Magnesium sulphate acts as membrane stabilizer (dose- $1 \mathrm{gm}$ iv stat then $1 \mathrm{gm}$ iv hrly for $3 \mathrm{hrs}$ then I gm continuous infusion daily for 4-7 days. The management of celphos poisoning is still supportive therapy. Gut decontamination especially if administered within 1-2 hrs, can be effective. Potassium permanganate $(1: 10,000)$ gastric lavage decomposes the toxin. The rationale behind use of mixture of soda bicarbonate and coconut oil with gastric lavage is guided by the chemical reaction of AlP with moisture and $\mathrm{HCl}$ to release phosphine gas, which rapidly gets absorbed by gastric mucosa. Soda bicarb helps in diluting the $\mathrm{H}$ Clther by inhibiting the breakdown of phosphide from pellets and Coconut oil provides a protective layer around the gastric mucosa, thereby preventing the absorption of phosphine gas.

The prognosis is based on various factors which include the manufacturing date of tablet, exposure of tablet prior to ingestion (old or new), duration of ingestion, arrival to hospital and initiation of management. ABG (severe acidosis), lactate levels guide for further management.

\section{Conclusion}

Since death is rapid and survival after significant poisoning is difficult, prevention is preferable. The most effective way of prevention is to either ban or impose strict regulations on sale of aluminium phosphide tablets. Shielding of tablets in smaller plastics with holes and spikes so that they can't be swallowed is likely to reduce the incidence. It is important that one must be aware of this poisoning as it is very easily available and extensively used. 